\title{
Retention of normal glia function by an isoform-selective protein kinase inhibitor drug candidate that modulates cytokine production and cognitive outcomes
}

Zhengqiu Zhou ${ }^{1 \dagger}$, Adam D. Bachstetter ${ }^{1,2,3 \dagger}$, Claudia B. Späni ${ }^{1}$, Saktimayee M. Roy ${ }^{4}$, D. Martin Watterson ${ }^{4}$ and Linda J. Van Eldik ${ }^{1,2,3^{*}}$

\begin{abstract}
Background: Brain p38a mitogen-activated protein kinase (MAPK), a potential therapeutic target for cognitive dysfunction based on the neuroinflammation-synaptic dysfunction cycle of pathophysiology progression, offers an innovative pharmacological strategy via inhibiting the same activated target in both glia and neurons, thereby enhancing the possibility for efficacy. The highly selective, brain-penetrant p38aMAPK inhibitor MW150 attenuates cognitive dysfunction in two distinct Alzheimer's disease (AD)-relevant models and avoids the problems encountered with previous mixed-kinase inhibitor drug candidates. Therefore, it is essential that the glial effects of this CNS-active kinase inhibitor be addressed in order to anticipate future use in clinical investigations.

Methods: We explored the effects of MW150 on glial biology in the AD-relevant APP/PS1 knock-in (KI) mouse model where we previously showed efficacy in suppression of hippocampal-dependent associative and spatial memory deficits. MW150 (2.5 mg/kg/day) was administered daily to 11-12-month-old KI mice for 14 days, and levels of proinflammatory cytokines IL-1 $\beta$, TNFa, and IL-6 measured in homogenates of mouse cortex using ELISA. Glial markers IBA1, CD45, CD68, and GFAP were assessed by immunohistochemistry. Microglia and amyloid plaques were quantified by immunofluorescence staining followed by confocal imaging. Levels of soluble and insoluble of $A \beta 40$ and $A \beta 42$ were measured by ELISA. The studies of in vivo pharmacodynamic effects on markers of neuroinflammation were complemented by mechanistic studies in the murine microglia BV2 cell line, using live cell imaging techniques to monitor proliferation, migration, and phagocytosis activities.

Results: Intervention with MW150 in KI mice during the established therapeutic time window attenuated the increased levels of IL-1 $\beta$ and TNFa but not IL-6. MW150 treatment also increased the IBA $1^{+}$microglia within a $15 \mu \mathrm{m}$ radius of the amyloid plaques, without significantly affecting overall microglia or plaque volume. Levels of IBA1, CD45, CD68, GFAP, and Aß40 and Aß42 were not affected by MW150 treatment. MW150 did not significantly alter microglial migration, proliferation, or phagocytosis in BV2 cells.

(Continued on next page)
\end{abstract}

\footnotetext{
* Correspondence: linda.vaneldik@uky.edu

${ }^{\dagger}$ Equal contributors

'Sanders-Brown Center on Aging, University of Kentucky, 800 S. Limestone

Street, Lexington, KY, USA

${ }^{2}$ Spinal Cord and Brain Injury Research Center, University of Kentucky, $741 \mathrm{~S}$.

Limestone Street, Lexington, KY, USA

Full list of author information is available at the end of the article
} 
(Continued from previous page)

Conclusions: Our results demonstrate that MW150 at an efficacious dose can selectively modulate neuroinflammatory responses associated with pathology progression without pan-suppression of normal physiological functions of microglia.

Keywords: Cytokines, Microglia, Protein kinase inhibitors, Mitogen-activated protein kinase 14, Neuroprotective agents, Alzheimer's disease

\section{Background}

Alzheimer's disease (AD) is the most common form of dementia, affecting $>5.3$ million people in the USA alone [1]. Current treatments stabilize symptoms temporarily without slowing progression of the disease [2]. Therefore, there is a pressing need for neuroprotective agents for $\mathrm{AD}$ and related dementias.

An array of complementary clinical and preclinical reports implicate discrete aspects of early stage neuroinflammation in disease progression. For example, genome-wide and genetic investigations reveal associations between inflammation-related genes and $\mathrm{AD}$ risk (for recent reviews, see [3-5]). Complementary to such associations are experimental outcomes from clinical pathology and animal model investigations that together provide a pathophysiology link between early stage neuroinflammation and AD risk (for reviews, see [6-8]). A specific form of neuroinflammation and dysregulated proinflammatory cytokine production, was shown to be a viable drug discovery pathway if intervention was in early stages when basal cytokine levels were progressively increasing prior to or coincident with synaptic dysfunction [9].

The p38 mitogen-activated protein kinase (MAPK) family of serine-threonine protein kinases, especially p38 $\alpha$ MAPK (MAPK14), are key regulators of proinflammatory cytokine production in the brain [10]. Activation of microglia p38 $\alpha \mathrm{MAPK}$ and the downstream overproduction of proinflammatory cytokines such as TNF $\alpha$ leads to synaptic protein loss, neurite degeneration, and neuronal death in microglia-neuron co-cultures [11]. Activation of p38 $\mathrm{MAPK}$ in neurons occurs in response to a variety of CNS disease-relevant stressors, and inhibition of

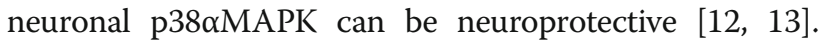
Taken in its entirety, the increasing body of evidence is consistent with activation of p38 $\alpha$ MAPK activity in both neurons and glia in response to disease-relevant stressors, raising the possibility that selective dosing with a brainpenetrant, isoform-selective p38 $\alpha$ MAPK could be a viable approach to disease modification or attenuation of disease progression susceptibility.

The recent description [14] and initial validation of a highly selective p38 $\mathrm{MMAPK}$ inhibitor, MW150, that is efficacious in two different AD models provided both a mechanistic precedent and a novel candidate for development.
Previous efforts at validating p38 $\alpha$ MAPK as a viable CNS target used mixed-kinase inhibitors whose results were difficult to interpret or lacked blood brain barrier penetrance and bioavailability to allow exposure to the brain-activated p38๙MAPK [15]. The development of MW150 solved those problems. Briefly, MW150 is a unique small molecule drug candidate that is the most highly specific p38 $\alpha \mathrm{MAPK}$ inhibitor reported to date. MW150 is orally bioavailable, CNS-penetrant, and efficacious in rescuing hippocampaldependent associative and spatial memory deficits in mouse models of AD-related pathology [14]. MW150 outcomes in a series of pharmacological screens forecast lower risk for development and documented the absence of safety problems that plagued previous CNS p38MAPK inhibitor campaigns. The battery of critical pharmacological and efficacy results qualified this unique, isoform-selective, p38 $\alpha$ MAPK inhibitor as a candidate for investigational new drug (IND) development required for first-in-human clinical studies. There is no comparable drug or drug candidate with the combination of documented specificity and safety, in vitro and in vivo pharmacological features, and target recognition $[14,15]$. Therefore, it is essential that the glial effects of this exceptional inhibitor with in vivo efficacy be addressed in order to anticipate future use in clinical investigations.

The goal of the current study was to determine in more depth the effects of MW150 on glial biology and proinflammatory cytokine dysregulation in the $\mathrm{AD}$ relevant APP/PS1 knock-in (KI) mouse model previously used to show MW150 efficacy in suppression of hippocampal-dependent associative and spatial memory deficits [14]. To further probe the selective action of MW150, we complemented the in vivo studies by live cell imaging analysis of the murine microglia BV2 cell line to monitor proliferation, migration, and phagocytosis activities. Our results demonstrate that MW150 has a selective role in modulation of neuroinflammatory responses without pan-suppression of normal physiological functions of microglia.

\section{Methods}

\section{Animals}

The AD mouse model we used is the $\mathrm{APP}^{\mathrm{NLh} / \mathrm{NLh}} \times$ PS1 ${ }^{\text {P264L/P264L }}$ KI mouse model originally developed at Cephalon [16]. This double KI mouse line expresses 
mutant APP and PS1 under the control of the endogenous promoters, and therefore, shows AD pathology without APP or PS1 overproduction. The APP/PS1 KI mice were maintained on a CD-1/129 background; wild-type (WT) control mice were obtained from heterozygous APP/PS1 matings and were maintained as a separate line for $>20$ generations, as previously described [9].

\section{Synthesis and use of MW150}

MW01-18-150SRM (MW150) was synthesized and characterized as previously reported [14]. For all experiments in this report, MW150 was dissolved in $0.9 \%$ sterile $\mathrm{NaCl}$ (saline: Hospira NDC 0409-4888-10) and was administered $(2.5 \mathrm{mg} / \mathrm{kg} /$ day) by intraperitoneal (i.p.) injection to 1112-month-ld APP/PS1 KI mice once daily for 14 days. APP/PS1 KI and WT mice administered saline vehicle i.p. under the same administration paradigm were used as controls.

\section{Brain tissue harvesting, biochemical, and immunohistochemical and immunofluorescent endpoints} Mice were anesthetized with $5 \%(\mathrm{v} / \mathrm{v})$ isoflurane prior to transcardial perfusion with ice-cold PBS for $5 \mathrm{~min}$. The brains were then rapidly removed and were fixed or homogenized as previously described [9]. IL-1 $\beta, T N F \alpha$, IL-6, A $\beta 40$, or $A \beta 42$ levels were measured in cortex homogenates using Meso Scale Discovery (MSD) ELISA, as previously described $[9,17,18]$. Immunohistochemical (IHC) staining was done, and images quantified with the Aperio ScanScope XT digital slide scanner and Aperio ImageScope software positive pixel count algorithm (version 9) as previously described [9, 17]. Primary antibodies used for IHC staining included: rabbit antiglial fibrillary acidic protein (GFAP) at 1:10,000 dilution (cat\# Z0334; Dako); rat anti-CD68 at 1:5000 dilution (cat\# MCA1957T; Serotec); and rat anti-CD45 (YW62.3) at 1:10,000 dilution (cat\# MA1447081; ThermoFisher Scientific). For the detection of GFAP, a HRP-conjugated goat anti-rabbit IgG was used. For all other primary antibodies, a biotinylated secondary antibody was amplified in avidin-biotin substrate ( $\mathrm{ABC}$ kit, Vector Laboratories). All sections were developed in $0.5 \mathrm{mg} / \mathrm{ml} \mathrm{3,3-diamino-}$ benzidine tetrahydrochloride solution (Sigma, cat\# D5637).

Immunofluorescence staining was done following established methods as previously described $[19,20]$. Antibodies used included: rabbit anti-IBA1 at 1:200 dilution (cat\# 019-19741; Wako); and biotin-labeled mouse anti-A $\beta$ (6E10) at 1:200 dilution (cat\# 39340-200, Covance). Primary antibodies were detected by Alexa 488 goat anti-rabbit IgG at 1:200 dilutions (cat\# A-11034, Life Technologies) or Alexa Fluor 594 streptavidin (cat\# S32356, Life Technologies). Immunofluorescent images were taken on a Nikon C2Plus Confocal Microscope using a $40 \times$ objective, at $18 \mu \mathrm{m}$ range with $0.175 \mu \mathrm{m}$ step size, $2 \times$ zoom, $512 \times 512$ pixel size, $0.0003 \mathrm{~mm} /$ pixel. Imaris software (version 8.1.2: Bitplane AG) was used for 3D reconstructions of the confocal Z-stacks. An observer blind to experimental conditions selected regions of interest in the cortex in a pseudo-randomized fashion using the presence of a $6 \mathrm{E} 10$ positive $A \beta$ plaque as the only criterion for selection. The surface creation tool was used to create surfaces for $A \beta$ and microglia. Amyloid plaque surfaces larger than 10,000 voxels were considered "large plaques". Distance transformation tool (MATLAB; version R2016b MathWorks) was used to create the distance channel from plaques. A surface of $15 \mu \mathrm{m}$ radius around large plaques was created using the distance channel. We empirically tested a range of different radiuses from $2-300 \mu \mathrm{m}$ on a test image. The goal was to find a radius that captured the majority of microglia that were plaque associated (i.e., touching), while avoiding microglia that were not in close proximity (i.e., not touching) the plaque. $15 \mu \mathrm{m}$ was chosen as the radius that best met these criteria.

\section{BV2 cell culture}

The murine microglial BV2 cell line [21] was cultured in DMEM/F12 media (cat\#15-090-CV, Mediatech) supplemented with $10 \% \mathrm{FBS}, 100 \mathrm{IU} / \mathrm{ml}$ penicillin, $100 \mu \mathrm{g} / \mathrm{ml}$ streptomycin (cat\# 30-002-CI, Mediatech), and $2 \mathrm{mM} \mathrm{L-}$ Glutamine (cat\# 25-005-CI, Mediatech), as previously described [18].

Proliferation, migration, and phagocytosis assays were done as previously described [19]. Cytochalasin D (CytD; cat\# C8273, Sigma), an inhibitor of actin polymerization, was used as a positive control. CytD was dissolved in dimethyl sulfoxide (DMSO; cat\# D2650, Sigma); therefore, a DMSO control at the same concentration was included in all experiments. As no difference was found between the saline control and the DMSO control, only the saline control values are shown.

For proliferation assays, BV2 cells were plated in a 96-well plate at 5000 cells/well in the presence of saline, DMSO $(0.01 \% \mathrm{v} / \mathrm{v})$, cytD $(1 \mu \mathrm{M})$, or MW150 (3.75, $7.5,15 \mu \mathrm{M})$. Cell density (image confluence) was recorded every $2-3 \mathrm{~h}$ using IncuCyte Zoom Live Cell Imager (Essen Bioscience) with 10x objective and analyzed with IncuCyte Zoom software (Essen Bioscience). Three independent experiments were performed, with eight technical replicates conducted for each experiment.

For phagocytosis assays, BV2 cells in a 96-well plate (5000 cells/well) were incubated with saline, DMSO $(0.01 \% \mathrm{v} / \mathrm{v})$, cytD $(1 \mu \mathrm{M})$, or MW150 $(3.75,7.5,15 \mu \mathrm{M})$ for 30 min. pHrodo red E. coli bioparticles (cat\# P35361, ThermoFisher Scientific) were then added to the wells at a final concentration of $400 \mu \mathrm{g} / \mathrm{ml}$. Fluorescence of the BV2 cells in the red channel was recorded 
every 30 min using IncuCyte Zoom at $20 \times$ objective. Three independent experiments were performed, with four technical replicates conducted for each experiment.

BV2 cell migration was assessed in a scratch wound assay. In a 96-well plate, the WoundMaker (Essen Bioscience) was used to create a strip devoid of cells in the center of each well when the cells were approximately $90 \%$ confluent. Saline, DMSO $(0.01 \% \mathrm{v} / \mathrm{v})$, cytD $(1 \mu \mathrm{M})$, or MW150 (3.75, 7.5, $15 \mu \mathrm{M})$ was added to each well, and images were recorded every $2-3 \mathrm{~h}$ using IncuCyte Zoom with $10 \times$ objective. The average size of the scratch wound that had filled with cells at $12 \mathrm{~h}$ post-scratch was determined by the percent confluency in the area left nearly devoid of cells after the scratch wound and normalized to vehicle. Three independent experiments were performed, with eight technical replicates conducted for each experiment.

MW150 inhibition of lipopolysaccharide (LPS)-induced proinflammatory cytokine upregulation in BV2 cells was measured as previously described [14]. Briefly, BV2 cells were plated at a cell density of $2 \times 10^{4}$ in a 48well plate and incubated for $24 \mathrm{~h}$. Cells were then treated with either saline control or $100 \mathrm{ng} / \mathrm{ml}$ of LPS (Salmonella enterica serotype typhimurium, cat\# L6143, Sigma, 600,000 EU/mg), in the absence or presence of increasing concentrations of MW150. After 16-h incubation, levels of TNF $\alpha$ in the conditioned media were measured by MSD ELISA.

\section{Statistics}

Graphs and statistical analyses were done using GraphPad Prism software version 6.0. A one-way analysis of variance (ANOVA) was used to compare three or more groups. A two-tailed Student's $t$ test was used for comparisons between $\mathrm{KI}+$ veh vs. KI + MW150-treated animals and WT + veh vs. KI + veh, as these comparisons were decided a priori to be the only ones of interest. A $p$ value $<0.05$ was considered significant. Values are expressed as mean \pm SEM. Data for all endpoints are available in Additional files 1, 2, 3 and 4: Tables S1-S4.

\section{Results}

MW150 reduces proinflammatory cytokine levels in the cortex of APP/PS1 KI mice

Previously, we found that MW150 rescued cognitive function as measured in the radial arm water maze in APP/PS1 KI mice [14]. Here, we investigated endpoints that may be associated with the protective effect of MW150 treatment in this mouse model. In many in vitro and in vivo systems, disease- or injury-induced activation of p38 $\alpha$ MAPK and subsequent upregulation of proinflammatory cytokines have been linked to downstream synaptic dysfunction. Therefore, the effect of MW150 treatment on proinflammatory cytokine levels was measured. For the study design, 11-12-month-old WT or KI mice were treated daily for 14 days with saline vehicle (veh) or MW150 (2.5 mg/kg) by i.p. injection. The mice were euthanized at day 19 after the start of treatment (Fig. 1a). As expected, based on our previous studies with the KI mice [9], protein levels of IL-1 $\beta$ in cortex homogenates were markedly elevated in $\mathrm{KI}+$ veh mice compared to WT + veh mice (Fig. 1b). MW150treated KI mice showed significantly reduced IL-1 $\beta$, with levels approaching those in WT mice (Fig. 1b). A similar pattern of changes was seen with TNFo; however, this change was not statistically significant (Fig. 1c). MW150 did not inhibit IL-6 levels under the conditions used in this experiment (Fig. 1d).

\section{MW150 treatment does not significantly alter GFAP immunostaining in the cortex of APP/PS1 KI mice}

To investigate whether astrocyte activation might be modulated by MW150 treatment, we measured a marker of reactive astrocytes, GFAP. The APP/PS1 KI mice showed higher levels of GFAP immunostaining than WT mice (Fig. 2a). KI mice treated with MW150 showed a trend of reduced GFAP staining in the cortex compared to $\mathrm{KI}+$ veh-treated mice, but this difference did not reach significance (Fig. 2b).

\section{MW150 treatment does not alter multiple markers of reactive microglia in the cortex of APP/PS1 KI mice}

IBA1 is a commonly used pan-marker of microglia and macrophages. Change in morphology (i.e., hypertrophy) of IBA1-positive cells is used as a marker of a reactive microglia response. To determine if MW150 had an effect on the reactive microglia response, we performed immunofluorescent staining for IBA1, followed by confocal microscopy and three-dimensional (3D) reconstruction using Imaris software. For each animal, 3-4 confocal z-stacks were collected. A 3D surface rendering was made of the IBA1 staining, and the total volume of the $3 \mathrm{D}$ z-stack that was occupied by the rendered $\mathrm{IBA} 1^{+}$ staining was determined, for approximately 40-70 microglia per animal. The volume of microglia staining included all $\mathrm{IBA}^{+}$staining captured in the z-stack including processes not associated with cell bodies. As shown in Fig. 3, there was no difference in the volume of microglia in the KI mice treated with MW150 or vehicle.

To further investigate changes in microglia/macrophage activation, we used two additional markers that are expressed at low levels in the mouse brain without an activating stimulus or pathology (Fig. 4). CD45 is a transmembrane protein that is expressed on all nucleated hematopoietic cells [22]. Its expression on resident microglia is low, but upon stimulation, its expression in microglia is upregulated [23]. IHC staining of CD $45^{+}$ 


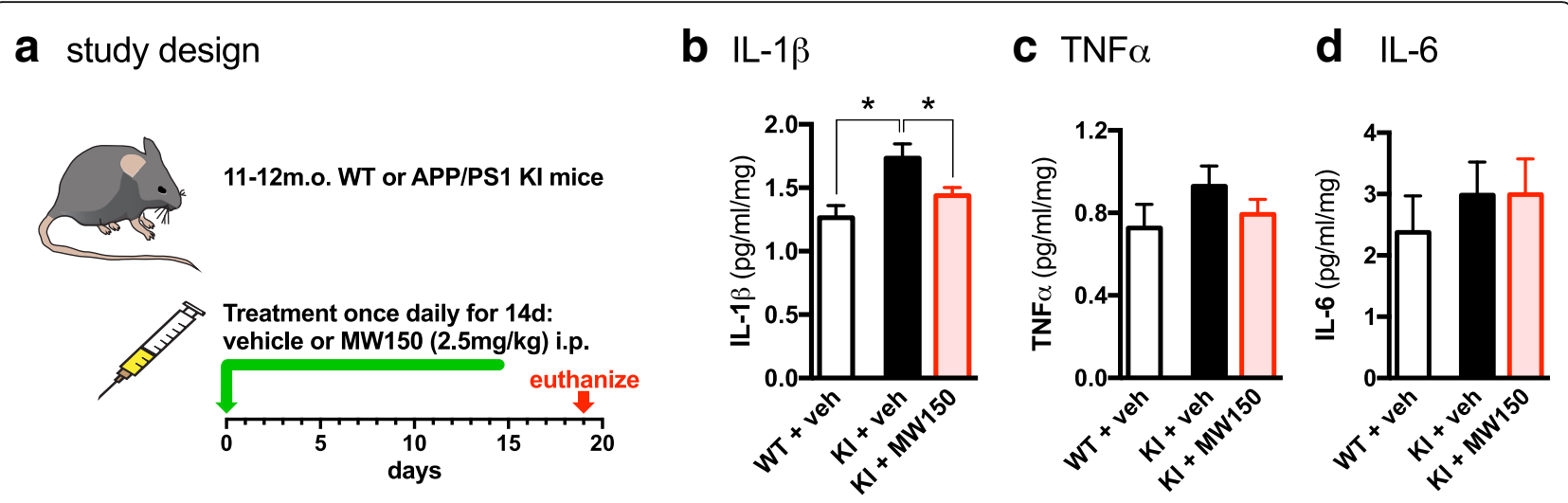

Fig. 1 Selective attenuation of proinflammatory cytokines by MW150 administration in APP/PS1 KI mice cortex. a 11-12-month-old wild type (WT) or APP/PS1 knock-in (KI) mice were treated with saline vehicle (veh) or $2.5 \mathrm{mg} / \mathrm{kg}$ MW150 by intraperitoneal injection (i.p.) once daily for 14 days. b IL-1 13 was increased in $\mathrm{Kl}$ + veh mice compared to $\mathrm{WT}+$ veh mice $(p=0.0012)$, and MW150 treatment of KI mice (KI + MW150) significantly attenuated IL-1 $\beta$ levels compared to $\mathrm{Kl}+$ veh $(p=0.0243)(F(2,38)=6.46 ; p=0.004)$. c TNFa was elevated in $\mathrm{Kl}+$ veh mice compared to $\mathrm{WT}+$ veh and attenuated in $\mathrm{Kl}+$ MW150 mice compared to $\mathrm{KI}+$ veh; however, these changes were not significant $(F(2,38)=1.11 ; p=0.34)$. $\mathbf{d} \mathrm{IL}-6$ was slightly elevated in $\mathrm{KI}+\mathrm{veh}$ compared to WT + veh mice, and there was no effect of MW150 treatment ( $n=11 \mathrm{WT}+$ veh; $n=14 \mathrm{KI}+$ veh; $n=14 \mathrm{KI}+\mathrm{MW} 150)$. Data are mean \pm SEM. Source data is available in Additional file 1: Table S1

cells in mice cortex was increased in KI mice compared to WT; however, $\mathrm{CD} 45^{+}$staining was not significantly altered with MW150 treatment (Fig. 4a. b). CD68 is a glycoprotein that is associated with lysosomes and is therefore linked to activated microglia, macrophages, and other phagocytic cells [24, 25]. CD68 was increased in the KI + veh mice compared to the WT mice, and MW150 treatment of the KI mice did not decrease CD68 staining (Fig. 4c, d).

\section{MW150 treatment has no significant effect on $A \beta$ plaque volume or on levels of soluble or insoluble $A \beta$ in the APP/PS1 KI mice}

We previously reported that MW150 had no effect on $A \beta$ plaque burden in the APP/PS1 KI mice [14], as assessed by
IHC staining. To test whether a potential effect of MW150 on $\mathrm{A} \beta$ might be revealed with quantitative and more detailed assays, we performed immunofluorescent staining and confocal analysis, as well as quantitative $A \beta$ ELISAs. $\mathrm{A} \beta$ plaques were stained with the $6 \mathrm{E} 10$ antibody, and microglia were stained with IBA1 (Fig. 5a). 3D reconstructions were generated from the confocal $z$-stacks using Imaris software, and $A \beta$ plaque volume was calculated. $A$ slight reduction was found in the total volume occupied by $\mathrm{A} \beta$ plaques in the $\mathrm{KI}+\mathrm{MW} 150$ compared to the $\mathrm{KI}+$ vehtreated mice, but the difference did not reach significance (Fig. 5b). Measurement of $A \beta 40$ and $A \beta 42$ levels in PBS soluble and formic acid (FA) soluble fractions of APP/PS1 KI mice cortex by quantitative A $\beta$ ELISA showed that MW150 had no effect on $A \beta$ levels (Fig. 5c).

\section{a GFAP in cortex}

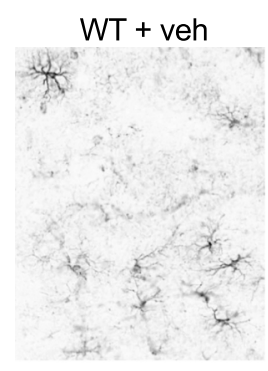

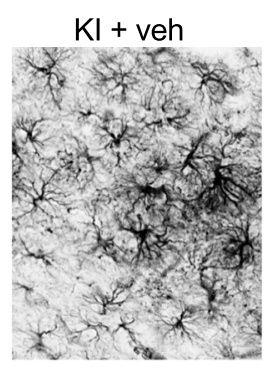

$\mathrm{KI}+\mathrm{MW} 150$

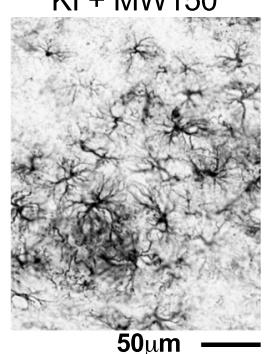

\section{b GFAP positive pixels}

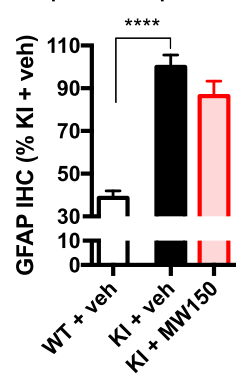

Fig. 2 No effect of MW150 on GFAP immunostaining. a Representative images of GFAP immunohistochemical (IHC) staining in cortex of WT or APP/PS1 KI mice treated with vehicle (veh) or MW150. b Digital quantification of GFAP in the cortex was done using the Aperio ScanScope with the entire cortex used as the region of interest. Quantification using the positive pixel algorithm showed a significant increase in GFAP staining in the $\mathrm{KI}+$ veh compared to $\mathrm{WT}+$ veh $(p<0.0001)$. No significant difference was found between the $\mathrm{KI}+$ veh compared to the $\mathrm{KI}+\mathrm{MW} 150$. $(\mathrm{F} 2,41)$ = 34.66; $p<0.0001)$. $(n=14 \mathrm{WT}+$ veh; $n=14 \mathrm{KI}+$ veh; $n=14 \mathrm{KI}+\mathrm{MW150})$. Data are mean \pm SEM. Source data is available in Additional file 2: Table S2 
a $\mid \mathrm{BA} 1^{+} 3 \mathrm{D}$ reconstruction

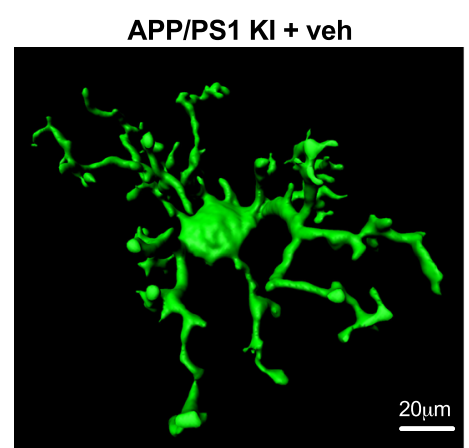

b IBA $1^{+}$area occupied

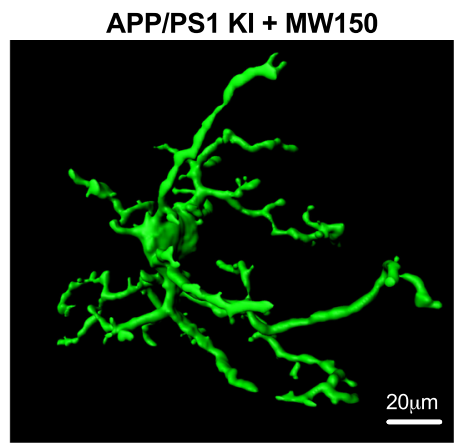

Fig. 3 No effect of MW150 on IBA $1^{+}$microglia volume. a Representative 3D surface reconstructions of IBA $1^{+}$cells generated from confocal microscopic imaging using Imaris software. $\mathbf{b}$ Mean volume \pm standard deviation (SD) of rendered IBA1 cells is shown for the APP/PS1 KI + veh and APP/PS1 KI + MW150 groups. Data represent mean of 3-4 independent z-stacks from each mouse. $(n=11 \mathrm{KI}+$ veh; $n=14 \mathrm{KI}+\mathrm{MW} 150)$

MW150 treatment increased plaque-associated IBA $1^{+}$cells but did not significantly increase $A \beta$ inside $I B A 1^{+}$cells in the cortex of $\mathrm{KI}$ mice

To determine if inhibition of p38 $\alpha$ MAPK would affect A $\beta$-microglial interactions, we tested the effect of MW150 treatment of KI mice on the number of microglia associated with amyloid plaques, and the amount of
$\mathrm{A} \beta$ internalized by microglia. To quantify the number of plaque-associated microglia, we generated a $3 \mathrm{D}$ reconstruction of $6 \mathrm{E} 10^{+}$amyloid plaques, with a focus only on $6 \mathrm{E} 10^{+}$amyloid plaques larger than 10,000 voxels. Using the distance transformation tool in the Imaris software, we then made a $3 \mathrm{D}$ region of interest ( $\mathrm{ROI}$ ) that was $15 \mu \mathrm{m}$ radius from the edge of the plaque and included a CD45 in cortex

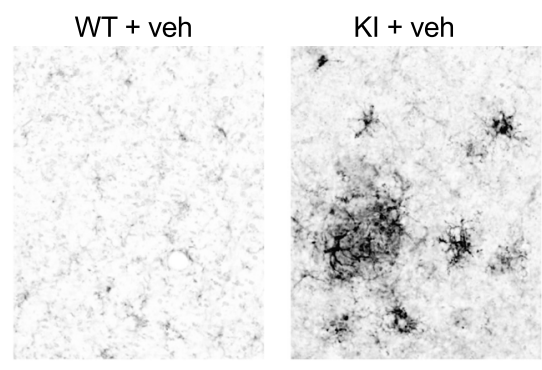

C CD68 in cortex

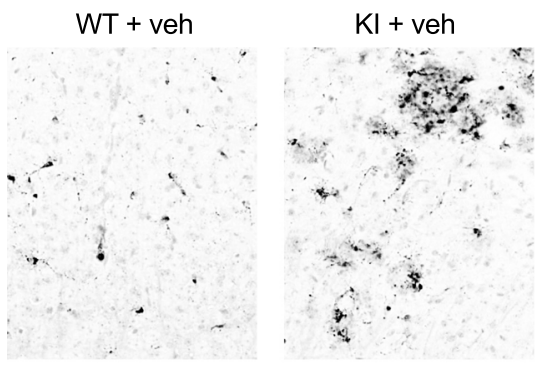

b CD45 positive pixels
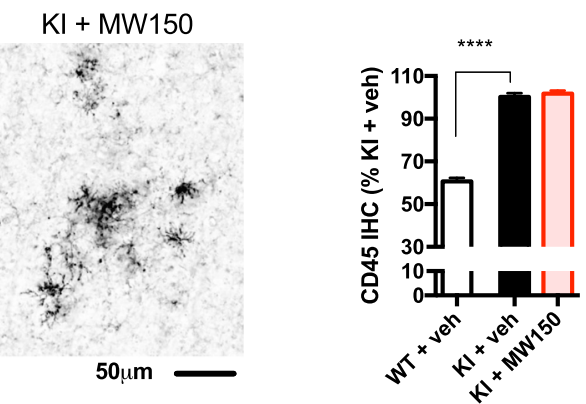

d CD68 positive pixels

\section{$\mathrm{KI}+\mathrm{MW} 150$}

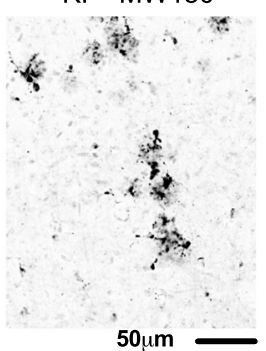

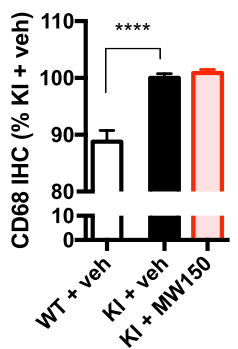

Fig. 4 No effect of MW150 on CD45 and CD68 immunostaining in the cortex. a Representative images of CD45 IHC in cortex of wild type (WT) or APP/PS1 KI mice treated with saline vehicle (veh) or MW150. Digital quantification of CD45 in the cortex was done using the Aperio ScanScope, and the positive pixel algorithm. $\mathbf{b}$ CD45 was significantly increased in the $\mathrm{Kl}$ + veh compared to the $\mathrm{WT}+$ veh treated mice $(p<0.0001)$, with no effect of MW150 treatment. $(F(2,42)=211.08 ; p<0.0001)$. c Representative images of CD68 IHC. d CD68 was significantly increased in the $\mathrm{Kl}+\mathrm{veh}$ compared to the WT + veh-treated mice $(p<0.0001)$, with no effect of MW150 treatment. $(F(2,42)=28.81 ; p<0.0001) .(n=14 \mathrm{WT}+$ veh; $n=14 \mathrm{Kl}+\mathrm{veh} ; n=14 \mathrm{Kl}+$ MW150). Data are mean \pm SEM. Source data is available in Additional file 2: Table S2 

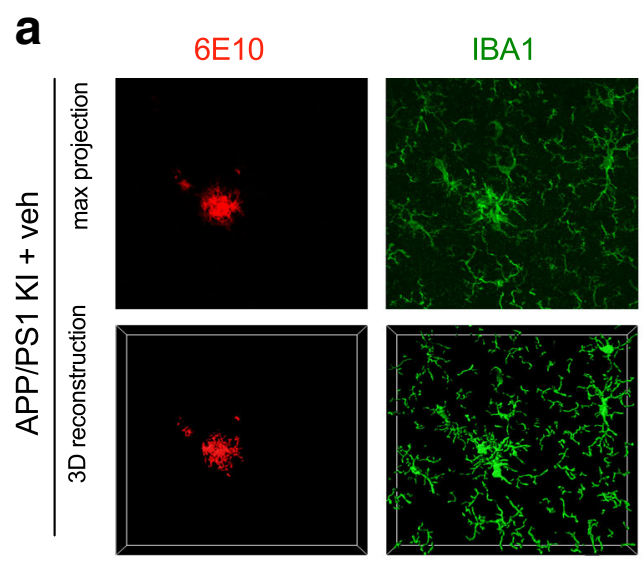

$6 \mathrm{E} 10 / \mathrm{IBA} 1$
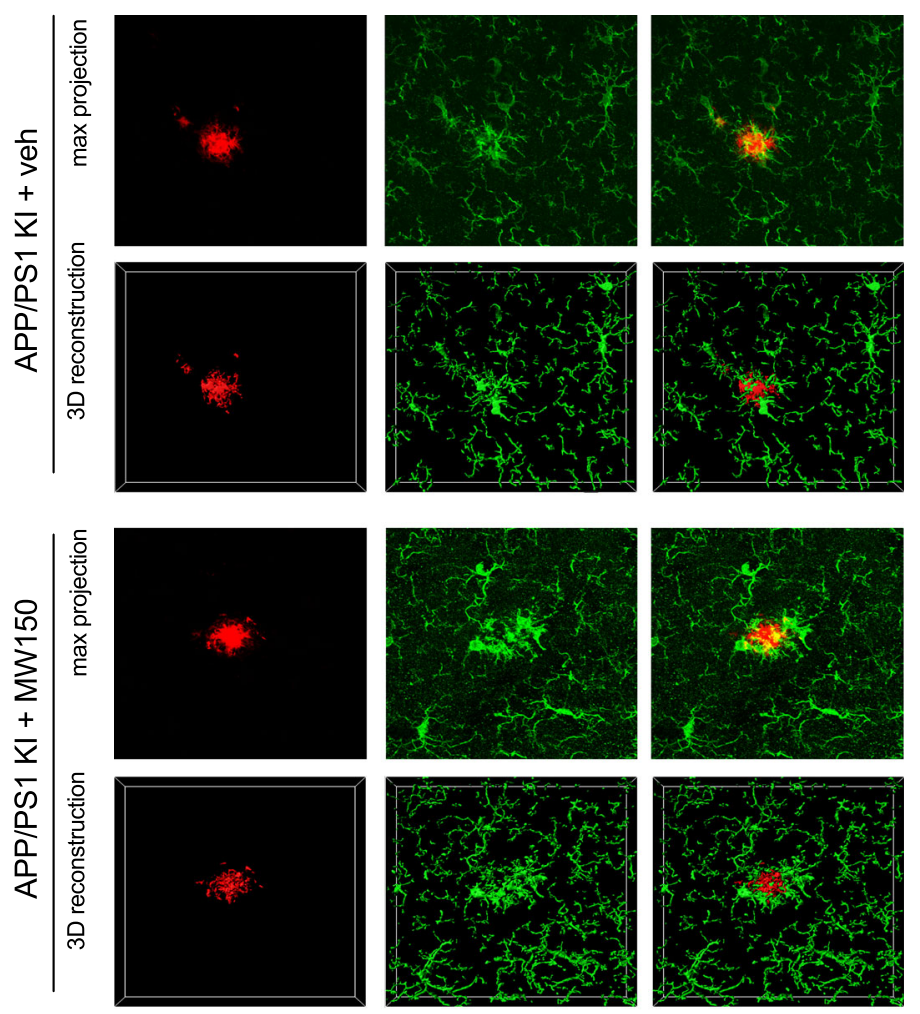

$50 \mu \mathrm{m}$
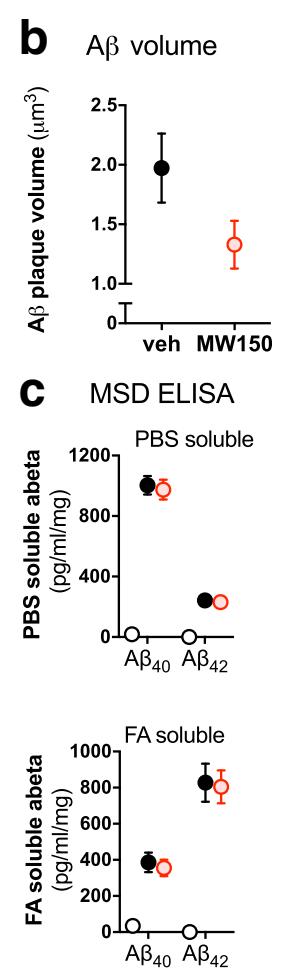

O $\mathrm{WT}+\mathrm{veh}$

APP/PS1 KI + veh

APP/PS1 KI + MW150

Fig. 5 No effect of MW150 on $A \beta$ in APP/KI mice. a $A \beta$ and microglia volume were measured using immunofluorescent staining with $6 E 10$ for $A \beta$ and IBA1 for microglia. A z-stack of images were taken using confocal microscopy then were analyzed using the surface tool in Imaris software. Representative confocal images and 3D surface reconstructions with Imaris software are shown. $\mathbf{b}$ A $\beta$ volume occupied by the surface reconstruction was reduced in $\mathrm{Kl}+\mathrm{MW150}$; however, the decrease was not significant. The data represents average of 3-4 independent z-stacks from each mouse $(n=11 \mathrm{Kl}+$ veh; $n=14 \mathrm{KI}+\mathrm{MW} 150)$. c PBS- and FA-soluble A 40 or A 42 levels were measured by MSD ELISA. No significant effect of MW150 treatment was found in the A $\beta$ ELISA. ( $n=11 \mathrm{WT}+$ veh; $n=14 \mathrm{KI}+$ veh; $n=14 \mathrm{KI}+$ MW150). Data are mean \pm SEM. Source data is available in Additional file 1: Table S1 and Additional file 3: Table S3

the plaque (Fig. 6a). Then, to determine the number of plaque-associated microglia, a surface rendering of the $\mathrm{IBA}^{+}$cell was generated (as in Fig. 3 ) for all of the $\mathrm{IBA} 1^{+}$staining in this 3D plaque-associated ROI. Quantification of the volume of $\mathrm{IBA}^{+}{ }^{+}$staining showed a significantly increased volume of $\mathrm{IBA}^{+}$staining surrounding each plaque in $\mathrm{KI}+\mathrm{MW} 150$ compared to the $\mathrm{KI}+$ veh-treated mice (Fig. 6b).

We further investigated whether MW150 had an effect on how much $A \beta$ was internalized by the $\mathrm{IBA}^{+}$cells. $\mathrm{A}$ mask for the $6 \mathrm{E} 10^{+}$staining was created, such that only $6 \mathrm{E} 10^{+}$staining that was colocalized with the surface rendering of the $\mathrm{IBA}^{+}$cells was included in the analysis (Fig. 6c). The volume of the $6 \mathrm{E} 10^{+}$staining within $\mathrm{IBA} 1^{+}$ staining was determined as a marker of the amount of $\mathrm{A} \beta$ internalized by the $\mathrm{IBA}^{+}{ }^{+}$cells. MW150 treatment resulted in a slight increase in the amount of $A \beta$ internalized by the $\mathrm{IBA}^{+}{ }^{+}$cells compared to $\mathrm{KI}+$ veh-treated mice, but the difference was not significant (Fig. 6d).

\section{MW150 treatment does not impair microglial} proliferation, migration, or phagocytosis in BV2 cells To further investigate the action of MW150 on microglia physiological functions, we used IncuCyte Zoom live cell imaging methods to measure proliferation, migration, and phagocytosis in a mouse microglial BV2 cell line exposed to increasing concentrations of MW150 (3.75 to $15 \mu \mathrm{M}$ ). Proliferation was measured by recording cell density (image confluence) at $30 \mathrm{~h}$ after compound treatment. Under the same culture conditions, we previously found [19] that BV2 cells show a linear cell growth rate at this time point. As shown in Fig. 7a, b, there was no significant effect of MW150 on BV2 cell density at any concentration tested. The positive control cytochalasin D (cytD), a known inhibitor of actin polymerization, caused a significant decrease in cell growth and an abnormal cell morphology (Fig. 7a, b).

Next, we assessed if MW150 would affect BV2 cell phagocytic ability, with an assay that uses E. coli 

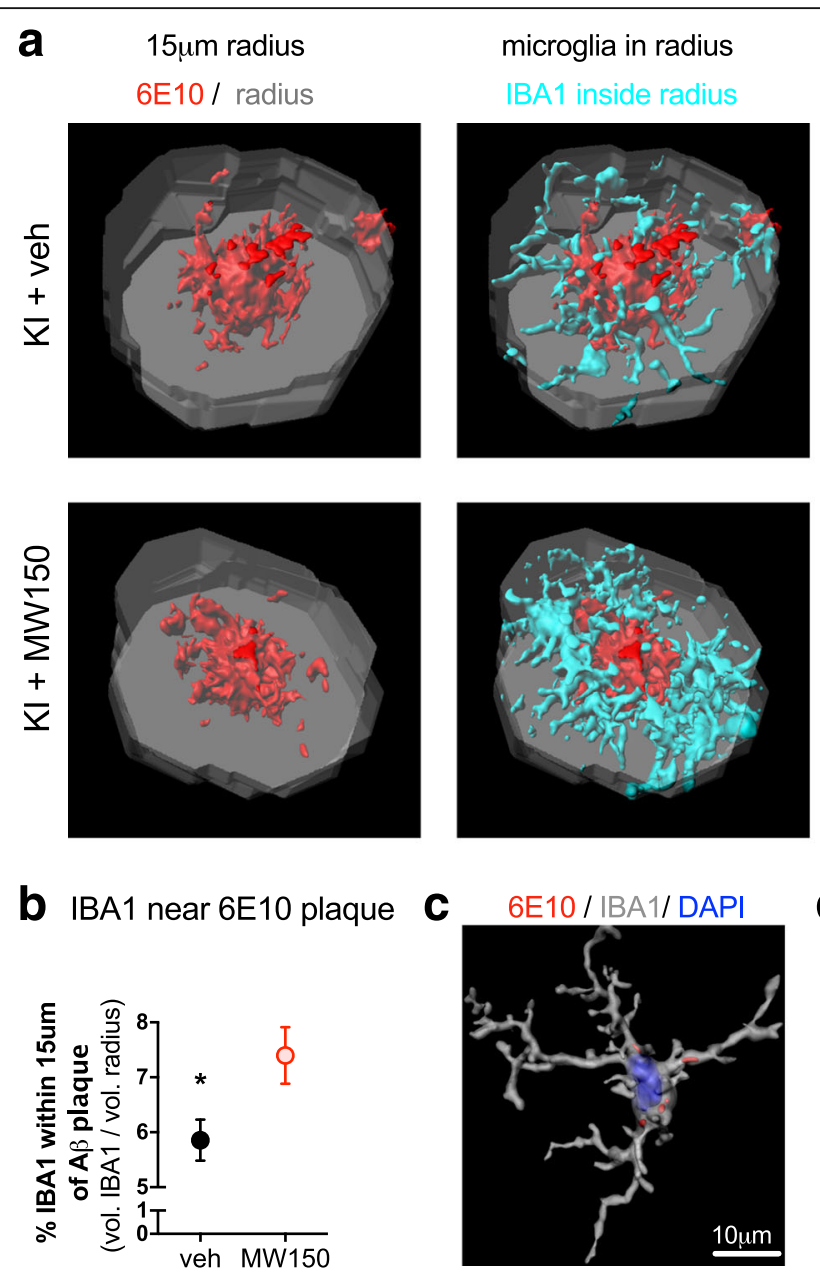

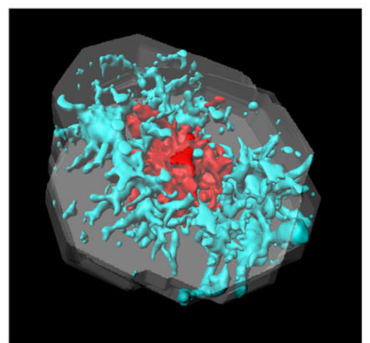

C

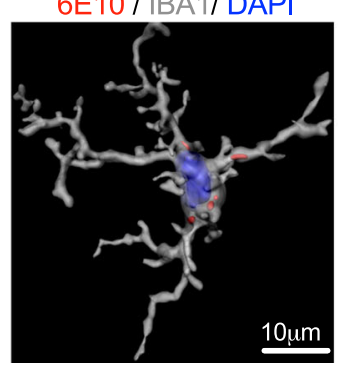

all microglia

IBA1 outside radius
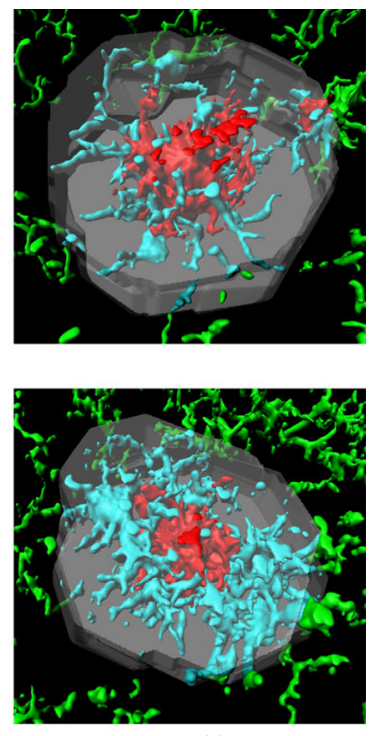

$20 \mu \mathrm{m}$

d $6 \mathrm{E} 10$ inside $\mathrm{IBA} 1^{+}$cell

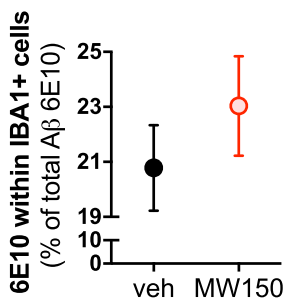

Fig. 6 Effect of MW150 treatment on microglia closely associated with A $\beta$ plaques and microglia-internalized A $\beta$ in the cortex of APP/PS1 KI mice. a Representative images of Imaris $3 \mathrm{D}$ reconstruction of plaques. A region of interest ( $R O$ ) was generated by expanding the plaque volume by a $15 \mu \mathrm{m}$ radius from the edge of the large plaques (larger than 10,000 voxels). This 3D ROI (shown in gray) included the A $\beta$ plaque, and a region near the plaque. IBA ${ }^{+}$cells in this ROI (shown in cyan) were surface rendered to create a 3D volume of all IBA1 positive staining in the ROI. The IBA1 positive staining in the 3D ROI distinguishes plaque-associated microglia (shown in cyan) compared to microglia away from plaques (shown in green). $\mathbf{b}$ Volume of surface rendered $\mid \mathrm{BA} 1^{+}$cells within $15 \mu \mathrm{m}$ radius around large plaques was significantly increased in $\mathrm{Kl}+\mathrm{MW} 150 \mathrm{mice}$ compared to $\mathrm{KI}+$ veh treatment $(p=0.0397)$. $\mathbf{c}$ Representative image of microglia reconstruction with DAPI stained nuclei showing 6 E 10 staining within $\mid \mathrm{BA} 1^{+}$cell cytoplasm. $\mathbf{d}$ Microglia-internalized $\mathrm{A} \beta$, as measured by $6 \mathrm{E} 10$ staining within surface rendered $\mathrm{IBA} 1^{+}$cells, was not significantly different between the $\mathrm{KI}+\mathrm{MW} 150$ compared to $\mathrm{KI}+$ veh. $(n=11 \mathrm{KI}+$ veh; $n=14 \mathrm{KI}+\mathrm{MW} 150)$. Data are mean \pm SEM. Source data is available in Additional file 3: Table S3

bioparticles as an indicator of phagocytosis. The bioparticles have a pHrodo dye that is non-fluorescent at neutral $\mathrm{pH}$ but becomes fluorescent in the red spectrum when it enters the acidic environment of a phagosome. MW150 $(3.75,7.5,15 \mu \mathrm{M})$ or CytD was added $30 \mathrm{~min}$ prior to addition of bioparticles, then fluorescence intensity was captured by IncuCyte Zoom software every $30 \mathrm{~min}$. We have previously reported [19] that in BV2 cells, the fluorescence intensity gradually increases over time and reaches a plateau by about 4 to $5 \mathrm{~h}$ after the addition of bioparticles. MW150-treated cells did not show significant differences in fluorescence intensity compared to saline-treated cells (Fig. 7c, d), but there was a trend toward increased phagocytosis with MW150. The positive control, CytD, significantly reduced the uptake of bioparticles by BV2 cells. The noticeable reduction in fluorescence intensity with CytD treatment is illustrated in Fig. 7d.

We used a scratch wound assay to assess the capability of BV2 cells to migrate into an injury area devoid of cell coverage. When BV2 cells reached approximately $90 \%$ confluency in a 96-well plate, a scratch wound was made by the Essen Bioscience WoundMaker. Previously we reported [19] that by $24 \mathrm{~h}$ the space made by the scratch would be filled 

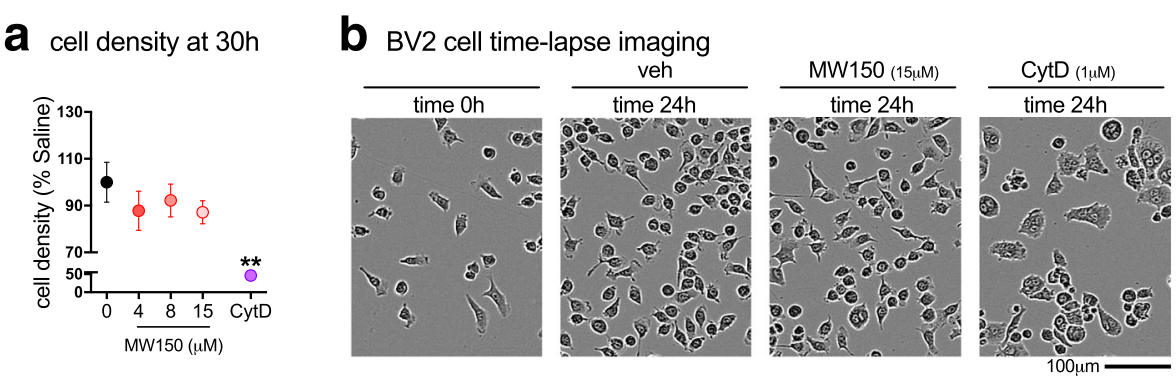

\section{C bioparticles at $3 \mathrm{~h}$}

d BV2 cell time-lapse imaging of bioparticle uptake
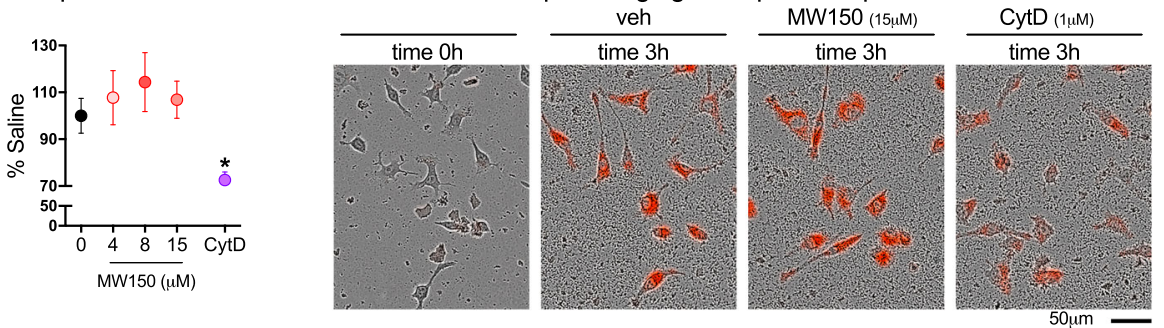

\section{e migration at $12 \mathrm{~h}$}

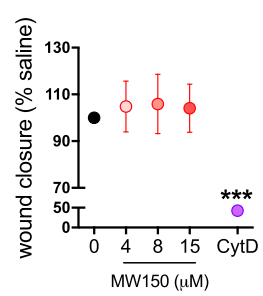

f BV2 cell time-lapse imaging

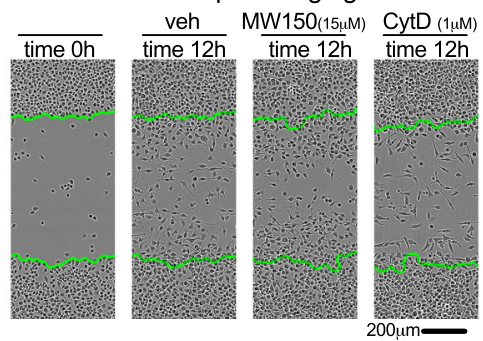

g MSD ELISA

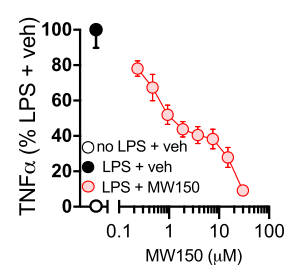

Fig. 7 No effect of MW150 treatment on BV2 cell proliferation, migration, and phagocytosis. a Average BV2 cell density at $30 \mathrm{~h}$ after plating in a 96-well plate at 5000 cells/well. b A representative example of the cell density at $24 \mathrm{~h}$ after treatment with saline, MW150 $(0,3.75,7.5$, or $15 \mu \mathrm{M})$, or cytochalasin $\mathrm{D}(\mathrm{cytD} ; 1 \mu \mathrm{M})$ (mean $\pm \mathrm{SEM}, n=3$ independent experiments; 8 technical replicates included for each experiment). c Quantification of pHrodo-labeled E. coli bioparticles at $3 \mathrm{~h}$ after addition of bioparticles. $\mathbf{d}$ A representative example of the cell bioparticles uptake at $3 \mathrm{~h}$ after treatment with saline, MW150 $(0,3.75,7.5$, or $15 \mu \mathrm{M})$, or cytD $(1 \mu \mathrm{M})$ (mean \pm SEM, $n=3$ independent experiments; 4 technical replicates included for each experiment). e Average size of scratch wound that is filled with cells, as determined by the percent confluency in the area left nearly devoid of cells after the scratch wound, normalized to veh at $12 \mathrm{~h}$ post scratch. $\mathbf{f}$ Representative images of the scratch wound made (highlighted by green lines), at time 0 and $12 \mathrm{~h}$ post scratch (mean \pm SEM, $n=3$ independent experiments; 8 technical replicates included for each experiment). g MW150 concentration-dependent inhibition of TNFa levels in LPS-stimulated BV2 cells (mean \pm SEM, $n=1-3$ independent experiments; 4 technical replicates included for each experiment). Source data is available in Additional file 4: Table S4

with BV2 cells. At $12 \mathrm{~h}$ after the scratch wound was made, MW150 treatment did not significantly alter the amount of empty space left by the scratch wound, whereas CytD caused a significant delay in wound closure (Fig. 7e, f).

Because MW150 had no suppressive activity in the proliferation, migration, or phagocytosis assays, it was important to confirm that MW150 was active in the cells. Therefore, as a positive control we tested the ability of MW150 to suppress TNF $\alpha$ production from LPSstimulated BV2 cells, a pathway known to be regulated by $\mathrm{p} 38 \alpha \mathrm{MAPK}$. We found that MW150 suppressed LPSstimulated TNFa upregulation with an IC50 of 1.34 to $1.99 \mu \mathrm{M}(95 \% \mathrm{CI})$, concentrations well below the range of MW150 (3.75, 7.5, $15 \mu \mathrm{M})$ used for the live cell imaging assays.

\section{Discussion}

There are two major findings in this study. First, treatment with an established efficacy dose of MW150 attenuated age-related increases in brain IL-1 $\beta$ and TNF $\alpha$ without affecting the overall amount of microglia or $A \beta$ levels. A surprising finding was that MW150 treatment increased the microglia closely associated with amyloid plaques. Second, MW150 treatment did not suppress protective microglia cell responses such as migration, proliferation, or phagocytosis over concentrations that attenuate proinflammatory cytokine production. These key findings document the selective effect of efficacious doses of MW150 on disease-linked glia processes with retention of responses considered protective.

The results reported here extend our previous validation [14] of MW150 as a candidate for development by 
showing that MW150 in the efficacious dose range suppresses IL-1 $\beta$ and TNF $\alpha$ overproduction in vivo in the APP/PS1 KI mice. We did not detect a change in IL-6 levels, consistent with the prevailing view that IL-6 cytokine induction generally occurs via signaling pathways independent of p38 $\alpha$ MAPK [26].

We previously reported [14] that MW150 showed no detectable effects on amyloid plaque burden in either the APP/PS1 KI mice or the APP/PS1 transgenic mice assessed by standard immunohistochemistry with $6 \mathrm{E} 10$ anti- $\mathrm{A} \beta$ antibody. We further extended the analyses here by using quantitative $A \beta$ ELISA and confocal microscopy. A $\beta$ ELISA demonstrated that MW150 had no detectable effect on the levels of PBS-soluble or formic acid-soluble $A \beta 40$ and $A \beta 42$ in the APP/PS1 KI mouse cortex, consistent with our previous study that reported no change in plaque load by immunohistochemistry [14]. Confocal microscopy analysis showed that MW150 treatment of APP/PS1 KI mice had no significant effect on the total $A \beta$ plaque volume in the cortex and on the amount of internalized $A \beta$ inside each microglia. However, even though the results did not reach significance, there was a trend toward decreased $A \beta$ plaque volume and increased $A \beta$ inside microglia in the APP/PS1 KI mice treated with MW150. These results are also consistent with a small increase in bioparticle phagocytosis activity seen in BV2 cells after MW150 treatment. Overall, our findings raise the intriguing possibility that MW150 stimulates microglia phagocytosis. However, these data must be interpreted with caution because the in vivo results with $A \beta$ plaque volume did not reach statistical significance, and in vitro results in the BV2 cell line with bioparticle phagocytosis cannot be directly equated to $A \beta$ phagocytosis in vivo. It will be important in future studies to test potential effects of MW150 on microglia phagocytosis in more detail. Nevertheless, it is clear that MW150 did not impair the ability of the plaque-associated microglia to phagocytose $A \beta$ and did not lead to a significant change in $A \beta 40$ or $A \beta 42$ levels.

An unexpected finding in the results reported here was that MW150 treatment significantly increased the microglia in close proximity to amyloid plaques. Whether this reflects an effect of MW150 on microglia migration is unclear. There was no statistically significant effect of MW150 on migration of BV2 cells in response to a scratch wound, but similar studies with migration to amyloid deposits were not done because of limited responsiveness of BV2 cells to synthetic $A \beta$. The functional significance of the increase in plaque-associated microglia is also not known, but a logical hypothesis to test in future studies is whether there is a relationship between modulation of p $38 \alpha \mathrm{MAPK}$ signaling, and the ability of microglia processes to surround amyloid plaques and promote their compaction. A demonstrated linkage would provide mechanisms whereby microglia could decrease compaction (p38 $\alpha$ MAPK mediated) or increase compaction (e.g., TREM2 mediated; [27]). The unexpected finding, therefore, raises the potential of subtle

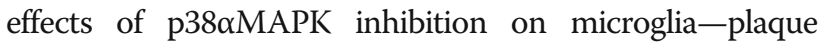
interactions that will require further exploration.

There is some general confusion in the literature about the pharmacodynamic effects of p38 $\alpha$ MAPK inhibitor drug candidates. Specific to this report, there is an unresolved issue in terms of the relationship between p38 $\alpha$ MAPK and amyloid pathology in preclinical mouse models of AD. Our results here and elsewhere [14] using multiple experimental approaches clearly show that the isoform-specific p38 $\alpha$ MAPK inhibitor MW150 does not significantly affect overall $A \beta$ levels or plaque burden. However, previous studies with some p38 $\alpha$ MAPK inhibitors that hit multiple kinases, such as VX-745 and CNI-1493, report inhibition of $\mathrm{A} \beta$ production or amyloid plaque deposition [28-30]. Whether the effects on these inhibitors on amyloid reflect engagement of targets other than p38 $\alpha$ MAPK [31, 32], such as Abl [33], is not known. Further, a full evaluation of the differences is limited by the type of animal model used in efficacy and pharmacodynamic endpoint analyses. For example, APP/PS1 transgenic mouse models that overexpress APP exhibit high levels of amyloid, whereas the APP/PS1 KI mouse uses endogenous promoters and demonstrates progressive $\mathrm{AD}$ pathology without the forced APP overproduction. Regardless, future clinical studies are required to resolve any therapeutic significance of these preclinical pharmacodynamic differences.

\section{Conclusions}

In summary, our results demonstrate the selective effects on glial inflammatory responses to treatment with MW150, a unique, isoform-selective, p38 $\alpha$ MAPK inhibitor drug candidate that attenuates cognitive impairment in AD-relevant mouse models. The findings show that MW150 has a selective role in modulation of neuroinflammatory responses without pan-suppression of normal physiological functions of microglia. The refined insight into glial biology presented here, combined with the safety and efficacy pharmacological profile of MW150 [14], reinforce the need to move this novel therapeutic candidate into clinical development for $\mathrm{AD}$ and related disorders.

\section{Additional files}

Additional file 1: Table S1. Cytokines and A $\beta$ MSD. Source data for cytokine data in Fig. 1 and A $\beta$ data in Fig. 5. (XLS 67 kb)

Additional file 2: Table S2. $I H C$ assays. Source data for GFAP data in Fig. 2 and CD68 and CD45 data in Fig. 4. (XLS 63 kb)

Additional file 3: Table S3. Confocal Imaris assays. Source data for microglia load in Fig. 2, plaque load in Fig. 5, and microglia/plaque data in Fig. 6. (XLS 59 kb)

Additional file 4: Table S4. BV2 assays. Source data for Fig. 7. (XLS 80 kb) 


\section{Abbreviations}

AD: Alzheimer's disease; ANOVA: Analysis of variance; CytD: Cytochalasin D; DMSO: Dimethyl sulfoxide; FA: Formic acid; GFAP: Glial fibrillary acidic protein; i.p.: Intraperitoneal; IHC: Immunohistochemical; KI: Knock-in; LPS: Lipopolysaccharide; MAPK: Mitogen-activated protein kinase; MSD: Meso Scale Discovery; MW150: MW01-18-150SRM; ROI: Region of interest; veh: Vehicle; WT: Wild-type

\section{Acknowledgements}

The authors thank Danielle Goulding for maintenance of the mouse colonies and excellent technical assistance.

\section{Funding}

This research was supported in part by NIH R01 NS093920 (LVE) and U01 AG043415 (DMW).

\section{Availability of data and materials}

All data generated or analyzed during this study are included in this published article and its supplementary information files.

\section{Authors' contributions}

ZZ participated in the design of the research studies, performed the confocal experiments and BV2 experiments, and prepared the first draft of the manuscript. ADB conceived the study, participated in the design and coordination, performed cytokine assays and histological assays, performed the statistical analyses, and participated in drafting the manuscript. CBS performed the confocal experiments, and participated in revising the manuscript. SMR contributed MW150, and participated in revising the manuscript. DMW participated in design of the study, contributed MW150, and participated in drafting the manuscript. LVE participated in the design of the study and its coordination, and participated in drafting the manuscript. All authors read, approved, and agreed to be accountable for all aspects of the final manuscript.

\section{Authors' information}

Not applicable.

\section{Competing interests}

DMW and LVE have a financial competing interest insomuch as they are principal investigators on project funding from the $\mathrm{NIH}$ and non-profit disease foundations that include the study of signal transduction pathways, drug target validation, or development of new molecular entities for treatment of CNS disorders; and Northwestern University and Columbia University have filed USA and international patent applications covering future commercial use of MW150. No non-financial conflicts of interest exist for any of the authors.

\section{Consent for publication}

Not applicable.

\section{Ethical approval and consent to participate}

All animal protocols followed the principles of animal care and experimentation in the Guide for the Care and Use of Laboratory Animals and were approved by the Institutional Animal Care and Use Committee of the University of Kentucky.

\section{Publisher's Note}

Springer Nature remains neutral with regard to jurisdictional claims in published maps and institutional affiliations.

\section{Author details}

${ }^{1}$ Sanders-Brown Center on Aging, University of Kentucky, 800 S. Limestone Street, Lexington, KY, USA. ${ }^{2}$ Spinal Cord and Brain Injury Research Center, University of Kentucky, 741 S. Limestone Street, Lexington, KY, USA. ${ }^{3}$ Department of Neuroscience, University of Kentucky, 800 Rose Street, Lexington, KY, USA. ${ }^{4}$ Department of Pharmacology, Northwestern University, 303 E Chicago Ave, Chicago, IL, USA.
Received: 13 January 2017 Accepted: 20 March 2017

Published online: 05 April 2017

\section{References}

1. Alzheimer's Association. Alzheimer's disease facts and figures. Alzheimers Dement. 2017;2017(13):325-73.

2. Pistollato F, Ohayon EL, Lam A, Langley GR, Novak TJ, Pamies D, Perry G, Trushina E, Williams RS, Roher AE et al. Alzheimer disease research in the 21 st century: past and current failures, new perspectives and funding priorities. Oncotarget. 2016;7(26):38999-39016.

3. Saykin AJ, Shen L, Yao X, Kim S, Nho K, Risacher SL, Ramanan VK, Foroud TM, Faber KM, Sarwar N, et al. Genetic studies of quantitative MCl and AD phenotypes in ADNI: Progress, opportunities, and plans. Alzheimers Dement. 2015;11(7):792-814.

4. International Genomics of Alzheimer's Disease C. Convergent genetic and expression data implicate immunity in Alzheimer's disease. Alzheimers Dement. 2015;11(6):658-71.

5. Benedet AL, Labbe A, Lemay P, Zimmer ER, Pascoal TA, Leuzy A, Mathotaarachchi S, Mohades S, Shin M, Dionne-Laporte A, et al. Epistasis analysis links immune cascades and cerebral amyloidosis. J Neuroinflammation. 2015;12:227

6. Wyss-Coray T, Rogers J. Inflammation in Alzheimer disease-a brief review of the basic science and clinical literature. Cold Spring Harb Perspect Med. 2012;2(1):a006346.

7. Van Eldik L, Carrillo MC, Cole PE, Feuerbach D, Greenberg BD, Hendrix JA, Kennedy M, Kozauer N, Margolin RA, Molinuevo JL, et al. The roles of inflammation and immune mechanisms in Alzheimer's disease. Alzheimers Dement. 2016;2(2):99-109.

8. Heppner FL, Ransohoff RM, Becher B. Immune attack: the role of inflammation in Alzheimer disease. Nat Rev Neurosci. 2015;16(6):358-72.

9. Bachstetter AD, Norris CM, Sompol P, Wilcock DM, Goulding D, Neltner $J H$, Clair DS, Watterson DM, Van Eldik LJ. Early stage drug treatment that normalizes proinflammatory cytokine production attenuates synaptic dysfunction in a mouse model that exhibits age-dependent progression of Alzheimer's disease-related pathology. J Neurosci. 2012; 32(30):10201-10.

10. Bachstetter AD, Van Eldik LJ. The p38 MAP kinase family as regulators of proinflammatory cytokine production in degenerative diseases of the CNS Aging Dis. 2010;1(3):199-211.

11. Xing B, Bachstetter AD, Eldik LJ. Microglial p38alpha MAPK is critical for LPSinduced neuron degeneration, through a mechanism involving TNFalpha. Mol Neurodegener. 2011;6:84.

12. Xing $B$, Bachstetter $A D$, Van Eldik $\sqcup$. Inhibition of neuronal p38alpha, but not p38beta MAPK, provides neuroprotection against three different neurotoxic insults. J Mol Neurosci. 2014;55(2):509-18.

13. Schnoder L, Hao W, Qin Y, Liu S, Tomic I, Liu X, Fassbender K, Liu Y. Deficiency of neuronal p38alpha MAPK attenuates amyloid pathology in Alzheimer disease mouse and cell models through facilitating lysosomal degradation of BACE1. J Biol Chem. 2016;291(5):2067-79.

14. Roy SM, Grum-Tokars VL, Schavocky JP, Saeed F, Staniszewski A, Teich AF, Arancio O, Bachstetter AD, Webster SJ, Van Eldik L, et al. Targeting human central nervous system protein kinases: an isoform selective p38alphaMAPK inhibitor that attenuates disease progression in Alzheimer's disease mouse models. ACS Chem Neurosci. 2015:6(4):666-80

15. Watterson DM, Grum-Tokars VL, Roy SM, Schavocky JP, Bradaric BD, Bachstetter AD, Xing B, Dimayuga E, Saeed F, Zhang H, et al. Development of novel in vivo chemical probes to address CNS protein kinase involvement in synaptic dysfunction. Plos One. 2013;8(6):e66226.

16. Flood DG, Reaume AG, Dorfman KS, Lin YG, Lang DM, Trusko SP, Savage MJ Annaert WG, De Strooper B, Siman R, et al. FAD mutant PS-1 gene-targeted mice: increased $A$ beta 42 and $A$ beta deposition without APP overproduction. Neurobiol Aging. 2002;23(3):335-48,

17. Bachstetter AD, Webster SJ, Goulding DS, Morton JE, Watterson DM, Van Eldik LJ. Attenuation of traumatic brain injury-induced cognitive impairment in mice by targeting increased cytokine levels with a small molecule experimental therapeutic. J Neuroinflammation. 2015;12:69.

18. Bachstetter AD, Xing B, de Almeida L, Dimayuga ER, Watterson DM, Van Eldik LJ. Microglial p38alpha MAPK is a key regulator of proinflammatory cytokine up-regulation induced by toll-like receptor (TLR) ligands or betaamyloid (Abeta). J Neuroinflammation. 2011;8:79. 
19. Bachstetter AD, Zhou Z, Rowe RK, Xing B, Goulding DS, Conley AN, Sompol P, Meier S, Abisambra JF, Lifshitz J, et al. MW151 inhibited IL-1 beta levels after traumatic brain injury with no effect on microglia physiological responses. Plos One. 2016;11(2):e0149451.

20. Bachstetter AD, Morganti JM, Jernberg J, Schlunk A, Mitchell SH, Brewster KW, Hudson CE, Cole MJ, Harrison JK, Bickford PC, et al. Fractalkine and CX 3 CR1 regulate hippocampal neurogenesis in adult and aged rats. Neurobiol Aging. 2011;32(11):2030-44.

21. Blasi E, Barluzzi R, Bocchini V, Mazzolla R, Bistoni F. Immortalization of murine microglial cells by a v-raf/v-myc carrying retrovirus. J Neuroimmunol. 1990; 27(2-3):229-37.

22. Penninger JM, Irie-Sasaki J, Sasaki T, Oliveira-dos-Santos AJ. CD45: new jobs for an old acquaintance. Nat Immunol. 2001;2(5):389-96.

23. Sedgwick JD, Schwender S, Imrich H, Dorries R, Butcher GW, ter Meulen V. Isolation and direct characterization of resident microglial cells from the normal and inflamed central nervous system. Proc Natl Acad Sci U S A. 1991:88(16):7438-42.

24. Kurushima H, Ramprasad M, Kondratenko N, Foster DM, Quehenberger O, Steinberg D. Surface expression and rapid internalization of macrosialin (mouse CD68) on elicited mouse peritoneal macrophages. J Leukoc Biol. 2000;67(1):104-8.

25. da Silva RP, Gordon S. Phagocytosis stimulates alternative glycosylation of macrosialin (mouse CD68), a macrophage-specific endosomal protein. Biochem J. 1999;338(Pt 3):687-94.

26. Heinrich PC, Behrmann I, Haan S, Hermanns HM, Muller-Newen G, Schaper F. Principles of interleukin (IL)-6-type cytokine signalling and its regulation. Biochem J. 2003;374(Pt 1):1-20.

27. Yuan P, Condello C, Keene CD, Wang Y, Bird TD, Paul SM, Luo W, Colonna M, Baddeley D, Grutzendler J. TREM2 haplodeficiency in mice and humans impairs the microglia barrier function leading to decreased amyloid compaction and severe axonal dystrophy. Neuron. 2016;90(4):724-39.

28. Bacher M, Dodel R, Aljabari B, Keyvani K, Marambaud P, Kayed R, Glabe C, Goertz N, Hoppmann A, Sachser N, et al. CNI-1493 inhibits Abeta production, plaque formation, and cognitive deterioration in an animal model of Alzheimer's disease. J Exp Med. 2008:205(7):1593-9.

29. Bach JP, Mengel D, Wahle T, Kautz A, Balzer-Geldsetzer M, Al-Abed Y, Dodel $\mathrm{R}$, Bacher M. The role of $\mathrm{CNI}-1493$ in the function of primary microglia with respect to amyloid-beta. J Alzheimers Dis. 2011;26(1):69-80.

30. Alam JJ. Selective brain-targeted antagonism of p38 MAPKalpha reduces hippocampal IL-1 beta levels and improves morris water maze performance in aged rats. J Alzheimers Dis. 2015;48(1):219-27.

31. Wang J, Grishin AV, Ford HR. Experimental anti-inflammatory drug semapimod inhibits TLR Signaling by targeting the TLR Chaperone gp96. J Immunol. 2016:196(12):5130-7.

32. Fabian MA, Biggs WH, Treiber DK, Atteridge CE, Azimioara MD, Benedetti MG, Carter TA, Ciceri P, Edeen PT, Floyd M, et al. A small molecule-kinase interaction map for clinical kinase inhibitors. Nat Biotechnol. 2005;23(3):329-36.

33. Cancino Gl, Toledo EM, Leal NR, Hernandez DE, Yévenes LF, Inestrosa NC, Alvarez AR. STI571 prevents apoptosis, tau phosphorylation and behavioral impairments induced by Alzheimer's $\beta$-amyloid deposits. Brain. 2008;131: 2425-42.

\section{Submit your next manuscript to BioMed Central and we will help you at every step:}

- We accept pre-submission inquiries

- Our selector tool helps you to find the most relevant journal

- We provide round the clock customer support

- Convenient online submission

- Thorough peer review

- Inclusion in PubMed and all major indexing services

- Maximum visibility for your research

Submit your manuscript at www.biomedcentral.com/submit

Biomed Central 